\title{
Validation of Random Positioning Versus Clinorotation Using a Macrophage Model System
}

\author{
Sonja Brungs ${ }^{1} \cdot$ Jens Hauslage ${ }^{1} \cdot$ Ruth Hemmersbach ${ }^{1}$
}

Received: 24 July 2018 / Accepted: 15 February 2019 /Published online: 2 March 2019

(C) The Author(s) 2019

\begin{abstract}
Clinostats and Random Positioning Machines (RPMs) are valuable devices for microgravity simulations in order to study fundamental gravity-dependent mechanisms on ground and in preparation for space flights. Both devices have different modes of operation, which have to be carefully considered and comprehensively discussed with respect to their potential impact on the quality of (simulated) microgravity. Here, we used the well-studied oxidative burst reaction of the immune cell (macrophage) model system, in order to compare clinorotation with random positioning. The RPM was used in a clinorotation mode, rotating the sample with $60 \mathrm{rpm}$ around the horizontal axis and in the "random speed mode" $(2-10 \mathrm{rpm})$ with and without random direction, thus, two axes rotating either bi- or unidirectionally. The production of Reactive Oxygen Species (ROS) during oxidative burst of the macrophages was visualized by the luminescence luminol assay. During clinorotation the cells responded with a reduction of the ROS production which is fully in line with earlier studies (clinostat, parabolic flight). In contrast, the exposure to the two random positioning modes showed that the oxidative burst response during RPM-exposure differs significantly from that observed during clinorotation, i.e. a jittering, more variant form of ROS production. We can conclude from our work, that the RPM is not suitable in its real random mode (with and without random direction; 2-10 rpm) to simulate the conditions of microgravity for the chosen system. We recommend that investigators using microgravity simulators should carefully choose the device and mode of operation specifically for their cellular system of interest.
\end{abstract}

Keywords Random positioning machine $\cdot$ Clinostat $\cdot$ Simulated microgravity $\cdot$ Macrophages

\section{Introduction}

To maintain human health during space flight, great effort has been made in the past years to study the effects of altered gravity on the immune system (Choukèr and Ullrich 2016). In preparation of experiments to be performed in microgravity and to increase the knowledge under normal gravity conditions, scientists design microgravity simulators (ground-based facilities) on which they expose their model system and study their question of interest. However, in order to validate the simulation approach, access to experiments in real microgravity are necessary,

Sonja Brungs

sonja.brungs@dlr.de

1 Institute of Aerospace Medicine, Gravitational Biology Department, German Aerospace Center (DLR), Linder Hoehe,

51147 Cologne, Germany but rare, especially those with identical set-ups as on ground in order to perform a perfect comparison.

Space immunology studies have focused on the influence of altered gravity on macrophages due to their role in immune response. Reactive Oxygen Species (ROS) are produced during their oxidative burst reaction, as the immunological firstline of defense and this is an easily measurable parameter of the immune response, therefore, perfectly suited for a space experiment. Several investigators have used chemoluminescence assays to quantify the amount of produced ROS in different cell types, in order to ascertain the influence of gravity on this crucial host-defense mechanism. Huber et al. (2006) established the methodology of luminol measurement in rodent alveolar macrophages, whereas Unruh et al. (2016) used the same assay to measure the ROS production in blue mussel hemocytes.

Immune cells produce ROS in terms of host-defense and the assay nicely demonstrates the activity and capability of the cells to fullfill their main duty i.e. clearance of the body after pathogen infection. This method has been adapted and 
improved in order to have a biomarker of immune cell activity under microgravity conditions (Adrian et al. 2013). Horn and colleagues developed a clinostat, capable of simulating microgravity on ground and in combination with a photomultiplier tube (PMT) to measure online the light emitted by the cells which is corresponding to the amount of ROS production (Horn et al. 2011). This breakthrough enabled real time measurements of ROS production in immune cells under altered gravity conditions and provided the ideal preparation for the TRIPLE LUX A \& B space experiments (Thiel et al. 2017; Ullrich et al. 2017; Unruh et al. 2016). Adrian et al. (2013) utilized the photomultiplier clinostat (PMT-clinostat) (Horn et al. 2011) to study the influence of simulated microgravity (clinorotation) on the ROS production. Furthermore, investigations included the application of hypergravity on a shortarm centrifuge as well as parabolic flight studies (Adrian et al. 2013; Brungs et al. 2015, 2016b). It was shown that the ground-based approaches using a clinostat as well as a centrifuge are excellent tools to simulate the conditions during a parabolic flight as the response was similar to the observed ROS production during parabolic flight (Brungs et al. 2016b). Several studies (Adrian et al. 2013; Brungs et al. 2015, 2016b) depicted the influence of altered gravity on the ROS production in alveolar macrophages and demonstrated that ROS production is a gravity-dependent process: microgravity revealed a decreased amount of ROS after cell stimulation, whereas hypergravity increased the production of radicals. Notably, the experiments were performed with the PMTclinostat and verified in real microgravity by exposure to parabolic flights (Adrian et al. 2013). The reaction of cells, in terms of the ROS production, is very fast and the underlying mechanisms remains to be studied, although, most likely the gravisensitivity remains within the phosphorylation of the crucial tyrosine kinase Syk (Brungs et al. 2015) or another fast responding process like the reorganization of the cytoskeleton or the Rho GTPase activity (Thiel et al. 2017). Taken together, these studies demonstrated that the pathogen-induced ROS production is a gravity-dependent process.

The aim of this study was to compare two different devices for microgravity simulation, the Random Positioning Machine (RPM) and the clinostat, with this well-studied assay. Clinostats rely on the principle of keeping the sample (cells) evenly suspended by constantly rotating the system to diminish sedimentation. The Random Positioning Machine is also used as a microgravity simulator, but is based on a different principle as compared to the 2D clinostat: the sample is rotated randomly around two axes of rotation, thereby the sample is continuously reoriented and - from the samples point of view the gravity trajectory is averaged over time to nearly zero (Mesland 1996; Brungs et al. 2016a). Until today, there is a number of literature available including studies with different microgravity simulators, ranging from free-fall machines, rotating-wall vessels, Random Positioning Machines to slow- and fast-rotating 2D/3D-clinostats (see review of Brungs et al. 2016a). Although clinostats have a long-lasting history and were validated for several model organisms, Random Positioning Machines gained popularity. High volumes of cell cultures and bulky containers are proposed to be used on the RPM, in contrast to small volumes and diameters suggested for 2D clinostats. Furthermore, more recently, RPMs have been successfully applied for tissue engineering studies, for example production of scaffold-free cartilage spheroids of thyroid cells and human chondrocytes (Grimm et al. 2014; Aleshcheva et al. 2016; Warnke et al. 2016). Several authors have stated that the best microgravity simulator has to be identified for each model system individually, depending on e.g. its sensitivity to accelerations and mechanical stress and cultivation conditions (Herranz et al. 2013). Therefore, there is a huge demand for comparative studies. Even more, since recent studies point out that random positioning results in the induction of fluid shear forces, which may affect the exposed cells and mask gravity-related effects (Wüest et al. 2015, 2017). Using the dinoflagellate Pyrocystis noctiluca and its capacity to act as bioassay for mechanical stress, Hauslage and coworkers visualized the shear forces induced by random positioning in contrast to clinorotation (Hauslage et al. 2017).

The macrophage test-system has been successfully employed on clinostats, centrifuges, parabolic flights and during space flight, thus, providing a robust test-assay, demonstrating online kinetics of ROS production as a marker for cellular activity and stress response. In the here presented study, we directly compared ROS production of macrophages, triggered by opsonized zymosan (yeast) particles, under different conditions - 2D-clinorotation as well as random positioning. This approach will increase our knowledge on the differences of these two kinds of simulation principles and support experimenters in choosing and discussing an appropriate method. Our overall goal is to provide information on the usability of microgravity simulators and to help investigators to design their experiments using ground-based facilities.

\section{Materials and Methods}

\section{Cell Cultivation and Luminol-Assay}

All experiments were carried out in the same design as done previously (Horn et al. 2011; Adrian et al. 2013; Brungs et al. 2015 , 2016b). Briefly, the alveolar macrophage cell line NR8383 (passages $<5$ ) was used for all experiments. Cells were cultivated under $95 \%$ relative humidity, $5 \% \mathrm{CO} 2$ at $37{ }^{\circ} \mathrm{C}$ in Ham's F12 (Biochrom), supplemented with $10 \%$ fetal calf serum (Biochrom) and $1 \%$ Antibiotic PenicillinStreptomycin (Biochrom), 0.1\% 2-Mercaptoethanol (Gibco). 
Medium was changed every 2-3 days. For each test assay $7 \times$ $10^{5} / \mathrm{ml}$ of cells in suspension was mixed in $1 \mathrm{ml}$ cuvette (diameter of $4 \mathrm{~mm}$ ) with horse radish peroxidase (500 units $/ \mathrm{ml}$; Merck) and $10 \mathrm{mM}$ luminol (AppliChem) in Borat buffer. To activate the cells, $100 \mu \mathrm{g} / \mathrm{ml}$ of opsonized zymosan (Sigma) was added and the measurement immediately started. The opsonized zymosan was prepared according to Allen (1986) and it was used as it initiates a strong oxidative burst in macrophages. Zymosan is phagocytosed by the cells and reactive oxygen species are produced, which, in turn, oxidize the added luminol solution. The conformational changes in the oxidized luminol results in the emittance of light, which can be measured/ amplified by a photon counting head equipped with a photomultiplier tube. For a non-activated control experiment, $10 \mu$ l of phosphate buffered saline (PBS; Biochrom) were added instead of zymosan. The PBS is only added to adhere to the procedure of adding a stimulus to the cells. PBS does not activate the cells. The measurement of ROS after addition of PBS (instead of zymosan) is the background ROS production; therefore this measurement can be seen as a negative control for the experimental set-up. Stressed cells or a high cell passage will show more background luminescence; therefore, this PBS control is very valuable as it indicates that the response is specific to the activation of the cells with zymosan.

\section{D Clinostat and Random Positioning Machine - Modes of Operation}

2D clinostats consist of a horizontally rotating sample cuvette, around one axis of rotation, with a small diameter and a rotating speed of $60 \mathrm{rpm}$ (Briegleb 1992). This rotation keeps the cells in suspension, as sedimentation is avoided and the gravity vector is continuously randomized (Brungs et al. 2016a). 2D clinostats are used for simulating microgravity for small biosystems such as cells, unicellular organisms, mussels, fish and others (Brungs et al. 2016a; Unruh et al. 2016).

Here, we used the "Desktop RPM" from Dutch Space (Leiden, Netherlands) as a 2D clinostat. The RPM consists of two frames which are positioned perpendicular to each other and can be rotated separately in several modes of operation. All experiments were performed on this kind of machine, however used in different modes: isolated rotation of the inner frame at $60 \mathrm{rpm}$, keeping the cell cuvette in a horizontal position. The cells were kept in a small cylindrical tube (cell culture volume $\sim 1 \mathrm{ml}$; diameter $4 \mathrm{~mm}$, length $3.5 \mathrm{~cm}$ ) and rotated in a horizontal position.

For 3D real random mode, samples are mounted on the inner frame and rotated around the two axes with randomly changing rotational speed and directions, with a speed varying between 2 and $85 \mathrm{rpm}$. In the present study two modes of operation were used: 1 . The "real random speed", where both frames operate at a maximum speed of $10 \mathrm{rpm}$ each at the same time in a constant direction. 2. The "real random speed" mode combined with "random direction" mode, in which both rotating frames are randomly changing the direction at a speed of maximum $10 \mathrm{rpm}$. The Desktop-RPM was located inside a cell culture incubator for all experiments.

The experimental set-up - implemented onto the inner frame of the RPM - was identical to the lately published hardware, described by Hauslage et al. (2017) (Fig. 1). The light emitted by the cells was measured by a digital photon counting head equipped with a photomultiplier tube (Hamamatsu, H7155). To reduce the amount of counted photons in a ratio of $4: 1$ and to amplify the electrical pulses a $74 \mathrm{HC} 193$ binary counter was implemented between the PMT and the Arduino Nano system. Obtained values were recorded by an Arduino program and saved on SD memory card as csv file. To perform a high rate counting of the Arduino up to $5 \mathrm{MHz}$ the FreqCounter library from Martin Nawrath from the Academy of Media Arts Cologne was used. The experiments were started with the addition of the

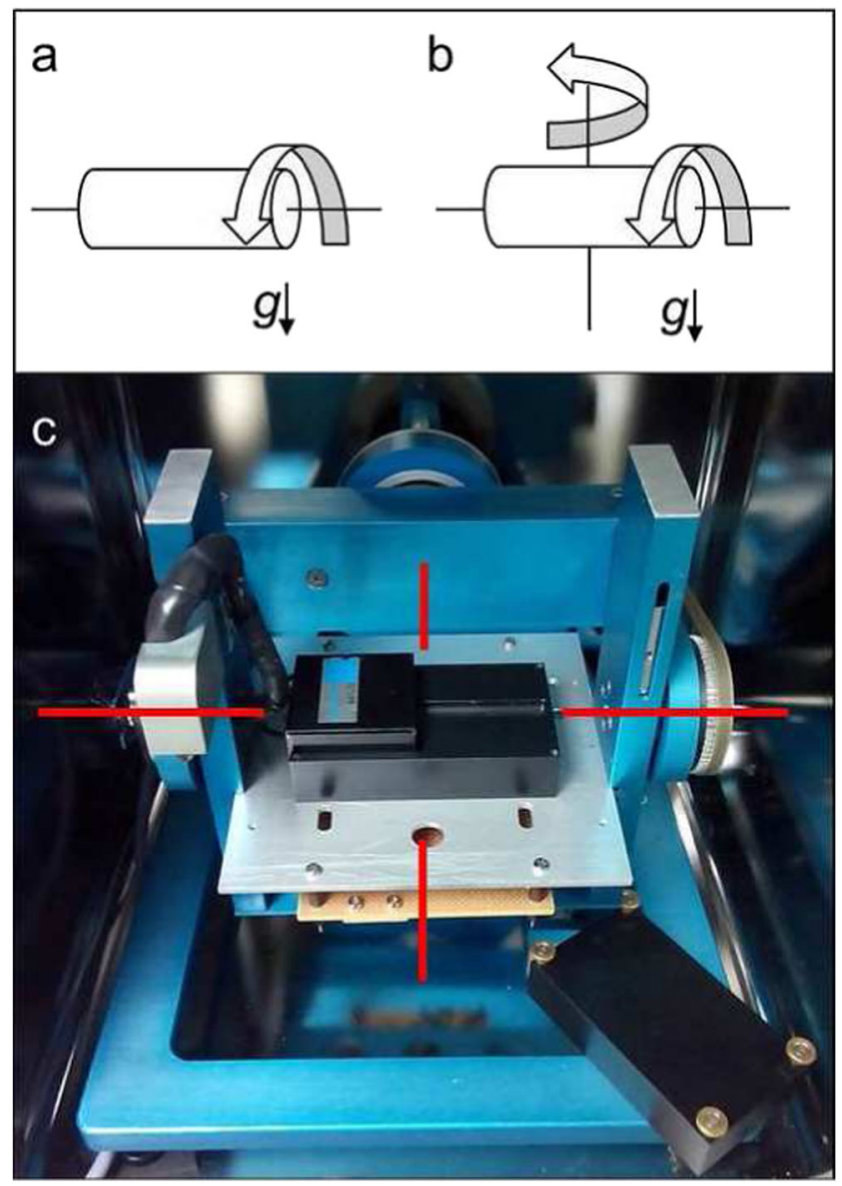

Fig. 1 The Random Positioning Machine set-up: a rotation of the inner axis as a clinostat with a speed of $60 \mathrm{rpm}$. b rotation of both axes with different speed and direction (real random mode with/without random direction). c The RPM set-up with the photomultiplier tube for bioluminescence measurements. Red lines indicate the different axes of rotation. Figure taken from Hauslage et al. 2017 with permission from the author 
oxidative burst-initiating zymosan and the measurements lasted for $45 \mathrm{~min}$, as this time frame is sufficient to record the major ROS production process. Experiments were repeated at least 6 times and the figures shown in the results section are representative for several experiments.

\section{Force Calculations}

In order to compare the clinostat mode with the random positioning mode, we calculated the peak (worst case) centrifugal force acting on a cell, assuming that the distance of a cell is maximal from the center of rotation.

The peak centrifugal acceleration applied to the system during clinorotation at $60 \mathrm{rpm}$ was calculated according to Wüest et al. (2015) by using the formula

$\mathrm{a}=\omega^{2} * \mathrm{r}$

$=\sim 0.08 \mathrm{~g}$ (constant value due to constant speed and direction of rotation)

With $\omega$ being the rotation velocity $(60 \mathrm{rpm} ; 6.283 \mathrm{rad} / \mathrm{s})$ and $r$ the inner radius of the cuvette in meters $(0.002 \mathrm{~m})$.

For RPM modes the force calculation is more difficult, because there are two frames rotated and the speed and direction is changed during the mode of operation. As an approximation we used 2.41, a number derived from calculation of the farthest position of the sample during rotation (see Wüest et al. 2014) and assuming both frames rotate constantly with a maximum speed of $10 \mathrm{rpm}(\sim 1.05 \mathrm{rad} / \mathrm{s})$ and a maximum radius from the center of rotation of $0.0175 \mathrm{~m}$ (length of cuvette is $3.5 \mathrm{~cm}$ ):

$\alpha=2.41 * \omega^{2} * \mathrm{r}$

$=\sim 0.05 \mathrm{~g}($ achieved over time due to random changes in speed and direction)

\section{Results}

In order to proof that the RPM is usable also as a clinostat, we rotated the inner frame of the RPM with $60 \mathrm{rpm}$ around its horizontal axis with the cell cuvette in the center of rotation, comparable to common 2D clinostats. Figure 2 shows the ROS production during one representative experiment of several runs $(N=6)$ using the RPM in the 2D clinostat mode. The ROS production, after stimulation with opsonized zymosan, is decreased during the clinorotation with its characteristic pattern: the ROS production under clinorotation is approximately $75 \%$ lower than the corresponding $1 \mathrm{~g}$ curve. Cells were used from different cell batches (passages) which results in a differing maximal luminescence and a high variation in the measured relative light units. The figures show the measurements from one batch and are representing measurements from several batches of cells. Furthermore, we performed control experiments using phosphate buffered saline (PBS) (instead of zymosan) as a negative control to see if vibrations etc. can induce a stress-related ROS production: The control experiment showed clearly that no ROS production was occurring.

Many investigators (Grimm et al. 2014; Hauslage et al. 2017; Wüest et al. 2017) used the RPM in the mode "real random speed" with and without "random direction" at a speed ranging between 2 and $10 \mathrm{rpm}$. We applied this operational program and monitored cellular ROS production visualized by the oxidation of luminol. A clear difference was stated compared to the pattern obtained in the clinostat experiments. In Fig. 3 it is shown how the RPM mode "real random speed" without "random direction" (unidirectional operation) produces very jittering curves with a high noise level. The same applies for the use of the real random mode with random direction as the mode produces jittering curves (Fig. 4). Taken together, RPM measurements significantly differ from the clinostat results: The cells produce (in total) less ROS during the exposure to random positioning (compared to $1 \mathrm{~g}$ control), but not as low as during clinorotation. Furthermore, a smooth curve as it is known from clinorotation was not detectable. Both RPM modes cause jittering ROS signals i.e. a more variant form of luminescence of higher and lower photon emissions, which is directly corresponding to the ROS production of the macrophages during the oxidative burst reaction. We used PBS instead of zymosan as a negative control, to elucidate whether the cells respond to the RPM modes with stressinduced ROS production, but hardly any ROS production occurred (compare Figs. 2 and 3).

\section{Discussion}

The aim of this study was to validate the usability of the RPM as a microgravity simulator for this specific cellular assay, which had been studied before using the PMT 2D clinostat technology (Horn et al. 2011). Before using the RPM modes of operation we set the speed of rotation of a single (inner) frame to $60 \mathrm{rpm}$ to have the same configuration as on a common clinostat. This was the perfect control experiment, as it provided the almost identical set-up to the RPM experiments itself. Rotating both frames of the RPM induces vibrations as shown by Krause et al. (2018). As a consequence, for clinorotation we used the inner frame which induces less vibration than the outer one. The data show that the ROS production is reduced in the $2 \mathrm{D}$ clinorotation mode, which is identical to the earlier finding using a PMT 2D clinostat (Horn et al. 2011; Adrian et al. 2013; Brungs et al. 2015). This experiment shows that the RPM device is in principle usable as a clinostat in case of macrophages, considering the sample is placed exactly in the center of the rotating axis and at a speed of $60 \mathrm{rpm}$. Furthermore, vibrations itself do not seem to induce any stress-related ROS production in macrophages - at least in a completely filled $1 \mathrm{ml}$ sample cuvette - as the control measurements with the by PBS non-activated cells did not show any response. 


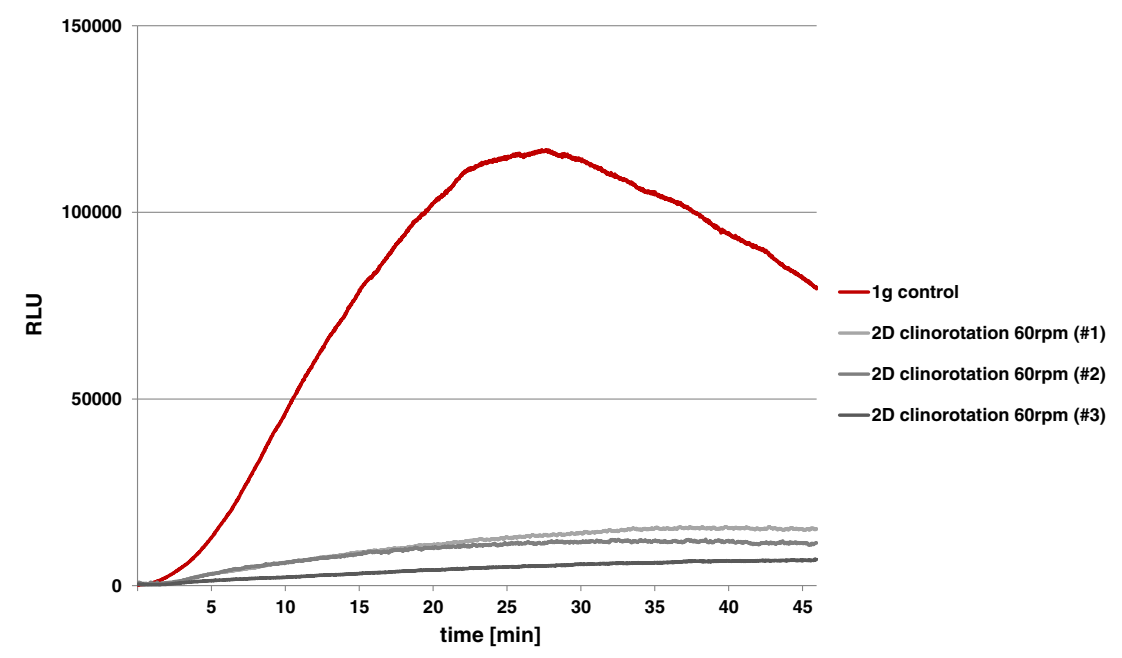

Fig. 2 2D clinorotation achieved on a Random Positioning Machine by just rotating one frame in a constant and fast mode $(60 \mathrm{rpm})$ reduces the ROS production of NR8383 macrophages after stimulation with $100 \mu \mathrm{g} / \mathrm{ml}$ opsonized zymosan (grey) compared to the stationary $1 \mathrm{~g}$

Both random RPM modes render the luminescence signal emitted by the cell, which significantly differs from the results of clinorotation. For the first time, such jittering, random ROS production has been observed using a microgravity simulator. Although the ROS curve maximum is lower than the respective $1 \mathrm{~g}$ control, random positioning does not provide a smooth curve and a drop in luminescence as shown during clinorotation.

\section{Macrophages Respond to Random Positioning}

Our findings do not indicate a difference between RPM modes with unilateral direction compared to random direction. Thus, control (red). The production of reactive oxygen species was visualized with the luminol-assay and a photomultiplier tube during clinorotation. Shown are individual measurements as representatives of several repetitions

we conclude that the macrophages are not influenced by the sudden changes in the direction when applying real random direction mode, but are influenced by random positioning itself. Our calculations show that the random positioning induced less centrifugal acceleration $(0.05 \mathrm{~g})$ compared to the clinorotation mode $(0.08 \mathrm{~g})$. Nevertheless, the cells respond with a completely different luminescence signal during random positioning compared to clinorotation. Here, we postulate that the cells are sensitive to the rotation of both frames together with the sudden change of direction of the rotation, although the theoretically resulting centrifugal force is lower than during clinorotation.

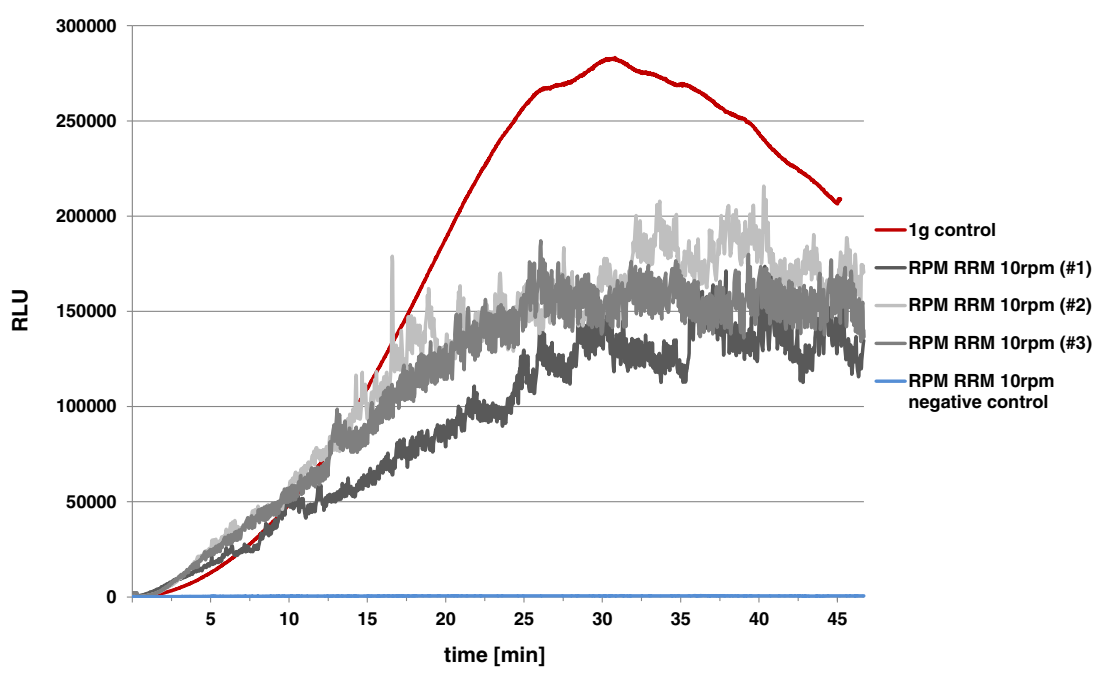

Fig. 3 RPM Real random speed mode at max. 10 rpm ("RPM RRM 10rpm"; grey) using 2 rotating frames at the same time renders the ROS production of NR8383 macrophages after stimulation with $100 \mu \mathrm{g} / \mathrm{ml}$ zymosan compared to the $1 \mathrm{~g}$ control (red). The non-activated cells activity (blue). The production of reactive oxygen species was visualized with the luminol-assay and a photomultiplier tube during random positioning. Individual measurements are shown as representatives of several repetitions 


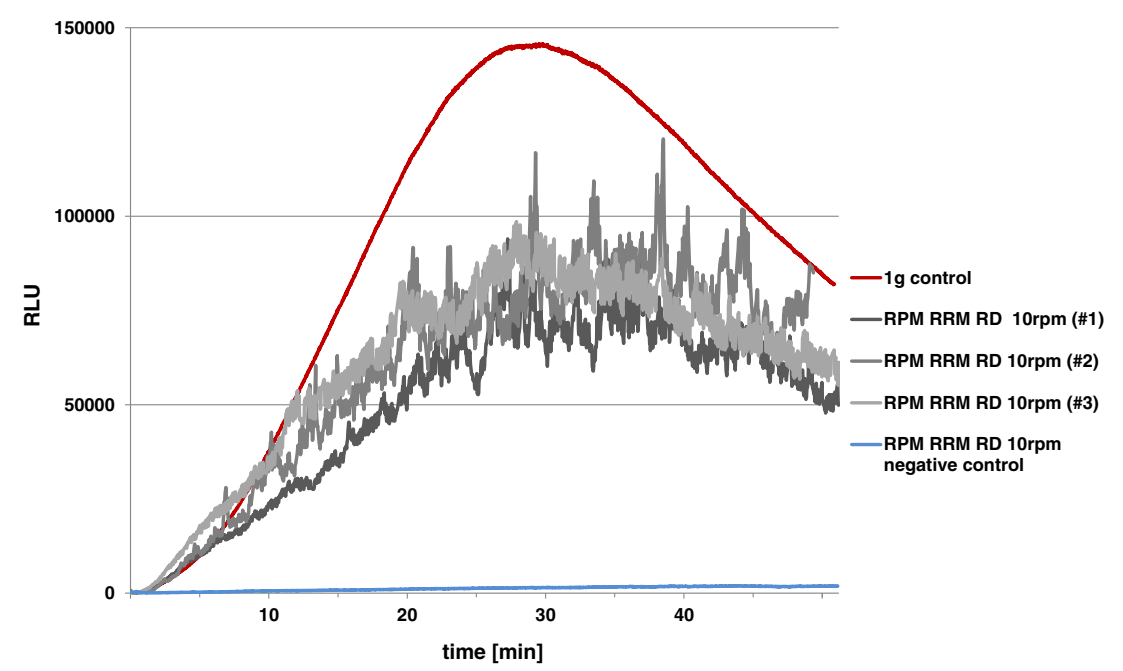

Fig. 4 RPM real random speed mode with random direction at max. $10 \mathrm{rpm}$ ("RPM RRM RD 10rpm"; grey) renders the ROS production of NR8383 macrophages after stimulation with $100 \mu \mathrm{g} / \mathrm{ml}$ opsonized zymosan compared to the $1 \mathrm{~g}$ control (red). The non-activated cells (zymosan replaced by PBS; "negative control") did not show any ROS activity (blue). The production of reactive oxygen species was visualized with the luminol-assay and a photomultiplier tube during random positioning. Individual measurements are shown as representatives of several repetitions
The principle of the RPM is to average the gravitational load to almost zero over time, meaning that mathematically zero gravity can be reached by running the RPM for a long period of time (which was calculated to be theoretically around $0.05 \mathrm{~g}$ ). Nevertheless, the actual gravity vector of all three dimensions varies between $-1 g$ and $+1 g$, which may influence the macrophages of the cell line NR8383. This cell line is known for its sensitivity to altered gravity as results from ROS measurements during parabolic flights showed that the cells respond to altered gravity/ changing gravity conditions within a few seconds (Adrian et al. 2013), indicating that this specific bioassay is capable of responding to changes in real-time. During the Triple Lux A experiment executed in the Columbus ISS facility Biolab, the macrophages responded to a sudden change from $1 \mathrm{~g}$ to $0 \mathrm{~g}$ with a sudden drop in luminescence, together with a fast adaptation to the microgravity environment within $42 \mathrm{~s}$ (Thiel et al. 2017).

\section{Cells May Respond to Shear Forces During Random Positioning}

In 2017, Wüest et al. (2017) modeled the shear stress occurring in the RPM during rotating of both frames with constant and equal velocity. Although their model included adherent cells in a bulky volume of a T75 cell culture flask - a set-up distinct from ours - we can conclude that the RPM induces shear forces. As shear forces on an RPM are most prominent in bulky containers with corners, we tried to keep the shear forces low, by using a cylindrical $1 \mathrm{ml}$ sample cuvette identical to the set-up in clinostat experiments, completely and bubble-free filled. The physical stimulation by shear forces may be sufficient to induce an altered ROS production in macrophages in case the cells were activated with a pathogen (zymosan) to trigger the oxidative burst pathway. It has been shown before, that the RPM can induce shear stress to unicellular organisms: The dinoflagellate Pyrocystis noctiluca was exposed to the identical experimental set-up showing a response to the shear forces during random positioning (Hauslage et al. 2017). As the presence of shear forces during RPM exposure were validated by computer simulations and using dinoflagellates as a reporter system (Hauslage et al. 2017; Wüest et al. 2017) we can conclude that the observed jittering luminescence and therefore radical production on the RPM operated in the mode random speed (2- $10 \mathrm{rpm})$ may be because of a sensitivity to shear forces. Notably, using PBS as a control "stimulant" we could not see any random ROS production of the cells which indicates that the jittering ROS production on the RPM after stimulation of zymosan is specific for the oxidative burst pathway.

Nevertheless, the underlying mechanism of this shearstress sensitivity of the macrophages remains elusive. The involvement of the key tyrosine kinase Syk (Brungs et al. 2015) may play a role in the gravisensitivity of the cells, however, it is also proposed that the cytoskeleton and NADPH oxidase and/or Rho GTPases may be involved (Thiel et al. 2017). We can speculate that these crucial proteins in ROS production pathway may also be affected by the conditions during random positioning. 
Although the production of luminescence in this macrophages system is different from the one occurring in Pyrocystis noctiluca, macrophages and dinoflagellates have something in common: in both systems, the origin of luminescence (radicals) is located at an organelle membrane. As Hauslage and colleagues proposed, altered gravity impacts the membrane fluidity, and, most likely, the signaling processes occurring on the membrane, resulting in altered luminescence in dinoflagellates (Hauslage et al. 2017) a phenomenon which could also be the reason for changed ROS production observed in macrophages.

\section{Conclusions and Recommendations Using the RPM}

Without the knowledge of the underlying mechanisms on how the change of gravity and/or shear-forces results in a change in the radical production, the here shown result cannot be fully interpreted. Nevertheless, the main purpose of this study was to use a well-studied model system in order to analyze the capabilities of the Random Positioning Machine as a microgravity simulator. We can conclude from our work, that the RPM is not suitable in its real random mode (with and without random direction; 2-10 rpm) to simulate the conditions of microgravity for the chosen system, as we were not able to reproduce the clinostat and parabolic flight results with the random positioning modes of the RPM. However, in its 2D clinostat mode, i.e. rotating the sample horizontally around just one axis with $60 \mathrm{rpm}$, the RPM can provide the same conditions as the previously validated PMT-clinostat (Horn et al. 2011). This has been also demonstrated in the case of statolith displacement in the rhizoid of Chara (Krause et al. 2018). Here, 2D clinorotation revealed comparable results as in real microgravity and thus an appropriate simulation. The additional rotation axis in 3D real random mode had no further advantage toward the 2D clinorotation with respect to the selected experimental parameters, but induced further vibrations (Krause et al. 2018).

Investigators using the RPM shall keep the volume of the sample container low and keep it in the center of rotation, in order to minimize shear force effects and gradients of accelerations. If applicable, users should make use of the clinostat mode (60 rpm, one axis of rotation) of the RPM in comparison to random positioning. Taken together, investigators shall carefully choose the microgravity simulator and try to compare RPM, clinostat and real microgravity (e.g. space flight) whenever possible, in order to validate the respective groundbased analogue and to avoid the induction of non-gravitational effects.
Acknowledgments We gratefully acknowledge the implementation of the PMT technology to the RPM by our colleague Kai Waßer.

We thank Simon Wüest for his inputs and discussion regarding the calculations of residual centrifugal forces.

Author's Contribution SB designed the study, executed all experiments and drafted the manuscript. JH designed, built and programmed the electronic hardware and edited the manuscript. RH edited the manuscript and discussed the experimental design and data.

\section{Compliance with Ethical Standards}

Competing Interests The authors declare no conflict of interest.

Open Access This article is distributed under the terms of the Creative Commons Attribution 4.0 International License (http:// creativecommons.org/licenses/by/4.0/), which permits unrestricted use, distribution, and reproduction in any medium, provided you give appropriate credit to the original author(s) and the source, provide a link to the Creative Commons license, and indicate if changes were made.

Publisher's Note Springer Nature remains neutral with regard to jurisdictional claims in published maps and institutional affiliations.

\section{References}

Adrian, A., Schoppmann, K., Sromicki, J., Brungs, S., von der Wiesche, M., Hock, B., Kolanus, W., Hemmersbach, R., Ullrich, O.: The oxidative burst reaction in mammalian cells depends on gravity. Cell Commun. Signal. 11, 98 (2013)

Aleshcheva, G., Bauer, J., Hemmersbach, R., Egli, M., Wehland, M., Grimm, D.: Tissue engineering of cartilage on ground-based facilities. Microgravity Sci. Technol. 28(3), 237-245 (2016). https://doi. org/10.1007/s12217-015-9479-0

Allen, R.: Phagocytic leukocyte oxygenation activities and chemiluminescence: a kinetic approach to analysis. Methods Enzymol. 133, 449-493 (1986)

Briegleb, W.: Some qualitative and quantitative aspects of the fastrotating clinostat as a research tool. ASGSB Bull. 5(2), 23-30 (1992)

Brungs, S., Kolanus, W., Hemmersbach, R.: Syk phosphorylation - a gravisensitive step in macrophage signaling. Cell Commun. Signal. 13(9), 9 (2015). https://doi.org/10.1186/s12964-015-0088-8

Brungs, S., Egli, M., Wüest, S.L., Christianen, P.C., van Loon, J.J., Anh, T.J.N., Hemmersbach, R.: Facilities for simulation of microgravity in the ESA ground-based facility programme. Microgravity Sci. Technol. 28(3), 191-203 (2016a). https://doi.org/10.1007/s12217015-9471-8

Brungs, S., Petrat, G., der Wiesche, M.V., Anken, R., Kolanus, W., Hemmersbach, R.: Simulating parabolic flight like g-profiles on ground - a combination of centrifuge and clinostat. Microgravity Sci. Technol. 28(3), 231-235 (2016b). https://doi.org/10.1007/ s12217-015-9458-5

Choukèr, A., Ullrich, O.: The Immune System in Space: Are we prepared? SpringerBriefs in Space Life Sciences. Springer International Publishing, Cham, s.l. (2016)

Grimm, D., Wehland, M., Pietsch, J., Aleshcheva, G., Wise, P., van Loon, J., Ulbrich, C., Magnusson, N.E., Infanger, M., Bauer, J.: Growing tissues in real and simulated microgravity: new methods for tissue engineering. Tissue Eng. B Rev. 20(6), 555-566 (2014)

Hauslage, J., Cevik, V., Hemmersbach, R.: Pyrocystis noctiluca represents an excellent bioassay for shear forces induced in ground- 
based microgravity simulators (clinostat and random positioning machine). npj Microgravity. 3(1), 1-7 (2017). https://doi.org/10. 1038/s41526-017-0016-x

Herranz, R., Anken, R., Boonstra, J., Braun, M., Christianen, P.C., de Geest, M., Hauslage, J., Hilbig, R., Hill, R.J., Lebert, M., Medina, F.J., Vagt, N., Ullrich, O., van Loon, J.J., Hemmersbach, R.: Ground-based facilities for simulation of microgravity: organismspecific recommendations for their use, and recommended terminology. Astrobiology. 13(1), 1-17 (2013). https://doi.org/10.1089/ast. 2012.0876

Horn, A., Ullrich, O., Huber, K., Hemmersbach, R.: PMT (Photomultiplier) Clinostat. Microgravity Sci. Technol. 23(1), 6771 (2011). https://doi.org/10.1007/s12217-010-9234-5

Huber, K., Krötz-Fahning, M., Hock, B.: Respiratory burst as a biomarker for stress responses. Protoplasma. 229(2-4), 221-224 (2006). https://doi.org/10.1007/s00709-006-0206-y

Krause, L., Braun, M., Hauslage, J., Hemmersbach, R.: Analysis of statoliths displacement in Chara rhizoids for validating the microgravitysimulation quality of clinorotation modes. Microgravity Sci. Technol. 30(3), 229-236 (2018). https://doi.org/10.1007/s12217017-9580-7

Mesland, D.A.: Novel ground-based facilities for research in the effects of weight. Microgravity News from ESA. 9, 5-10 (1996)

Thiel, C.S., Zelicourt, D., de Tauber, S., Adrian, A., Franz, M., Simmet, D.M., Schoppmann, K., Hauschild, S., Krammer, S., Christen, M., Bradacs, G., Paulsen, K., Wolf, S.A., Braun, M., Hatton, J., Kurtcuoglu, V., Franke, S., Tanner, S., Cristoforetti, S., Sick, B., Hock, B., Ullrich, O.: Rapid adaptation to microgravity in mammalian macrophage cells. Sci. Rep. 7(43), 1-13 (2017). https://doi.org/ 10.1038/s41598-017-00119-6
Ullrich, O., Hock, B., Thiel, C.: The preparation of the space trial TRIPLE LUX A through hardships to the stars. Flugmedizin, Tropenmedizin, Reisemedizin. 24(4), 201-205 (2017)

Unruh, E., Brungs, S., Langer, S., Bornemann, G., Frett, T., Hansen, P.D.: Comprehensive study of the influence of altered gravity on the oxidative burst of mussel (Mytilus edulis) hemocytes. Microgravity Sci. Technol. 28(3), 275-285 (2016). https://doi.org/10.1007/ s12217-015-9438-9

Warnke, E., Kopp, S., Wehland, M., Hemmersbach, R., Bauer, J., Pietsch, J., Infanger, M., Grimm, D.: Thyroid cells exposed to simulated microgravity conditions - comparison of the fast rotating clinostat and the random positioning machine. Microgravity Sci. Technol. 28(3), 247-260 (2016). https://doi.org/10.1007/s12217-015-9456-7

Wüest, S.L., Richard, S., Walther, I., Furrer, R., Anderegg, R., Sekler, J., Egli, M.: A novel microgravity simulator applicable for threedimensional cell culturing. Microgravity Sci. Technol. 26(2), 7788 (2014)

Wüest, S.L., Richard, S., Kopp, S., Grimm, D., Egli, M.: Simulated microgravity: critical review on the use of random positioning machines for mammalian cell culture. Biomed. Res. Int. 971474. 2015, 1-8 (2015). https://doi.org/10.1155/2015/971474

Wüest, S., Stern, P., Casartelli, E., Egli, M.: Fluid dynamics appearing during simulated microgravity using random positioning machines. PLoS ONE. 12(1), 1-19 (2017). https://doi.org/10.6084/m9. figshare. 4555852 